\title{
Modelo de "análisis de resultados del estado actual" (AREA) en un paciente con VIH/SIDA para el plan de cuidados de enfermería. Caso clínico
}

\author{
"Current state results analysis" (CSRA) model in a patient with HIVIAIDS for the \\ nursing care plan. Case report
}

\author{
Adrián Daniel Carrera-Cisneros ${ }^{1}$ y Patricia Larios-López ${ }^{2}$ \\ 1 Alumno de la Carrera Enfermería, Facultad de Estudios Superiores Zaragoza, UNAM \\ 2 Profesora de asignatura de la carrera de Enfermería, Facultad de Estudios Superiores Zaragoza, UNAM
}

\begin{abstract}
ResUMEN
Introducción. Las personas con VIH/SIDA presentan varios síntomas relacionados con la enfermedad, efectos secundarios a los antiretrovirales y múltiples complicaciones. El régimen terapéutico es complejoy requiere un diagnóstico preciso y de los cuidados de enfermería. En este sentido, una de las opciones para dicho fin es la aplicación del modelo de análisis de resultados del estado actual (AREA). Por tal motivo, se presenta un análisis crítico de un caso clínico de un paciente con VIH/SIDA con múltiples patologías en el que se presenta la reseña de plan de cuidados de enfermería convencional y se contrasta con la aplicación del modelo AREA empleando en el trabajo en red para visualizar el diagnóstico con un enfoque holístico. Casoclínico. Paciente masculino de 57 años, con diagnóstico médico principal de $\mathrm{VIH}$, hepatitis $\mathrm{B}$, diabetes mellitus tipo 2, infarto agudo al miocardio de antaño y una colecistectomía crónica litiasica actual que complica el estado de salud del paciente. Se realizó un análisis crítico del caso utilizando el métodoAREA, para un razonamiento diagnóstico en red, mediante el cual se identificó 3 diagnósticos enfermeros prioritarios: gestión ineficaz de la salud, protección ineficaz y disminución del gasto cardiaco con la formulación de resultados esperados e intervenciones de enfermería. Conclusión. Las mejores prácticas clínicas de enfermería son fundamentales para la calidad de la atención y el confort de los pacientes, especialmente en diagnósticos complejos de polipatología como son los casos de pacientes no controlados con $\mathrm{VIH} /$ SIDA, porloqueel modeloAREAes una buenaopción, yaque promueve la reflexión analítica y crítica de los profesionales de enfermería para el diagnóstico integral y holístico.
\end{abstract}

Palabras clave: $\mathrm{VIH} / \mathrm{SIDA}$, cuidados de enfermería, método AREA.

\begin{abstract}
Introduction. People with HIV / AIDS have several symptoms related to the disease, side effects of antiretrovirals, and multiple complications. The therapeutic regimen is complex and requires an accurate diagnosis and nursing care. In this sense, one of the options for this purpose is the application of the current state results analysis model (CSRA). For this reason, a critical analysis of a clinical case of a patient with HIV / AIDS with multiple pathologies is presented. Here, the conventional nursing care plan is reviewed, featured and contrasted with the application of the CSRA model, using a network to visualize the diagnosis under a holistic approach. Clinical case. A 57-year-old male patient with a primary medical diagnosis of HIV, hepatitis B, type 2 diabetes mellitus, an acute myocardial infarction of the past and a current chronic lithiasic cholecystectomy that complicates the patient's health status. A critical analysis of the case was carried out using the CSRA method, for a networked diagnostic reasoning, through which three priority nursing diagnoses were identified: ineffective health management, ineffective protection and decrease in cardiac output with the formulation of expected results and nursing interventions. Conclusion. The best clinical nursing practices are fundamental for the patients care and comfort quality, especially in complex polypathology diagnoses such as the cases of uncontrolled patients with HIV / AIDS. Hence, the CSRA model is a good option, since it promotes analytical and critical reflection of nursing professionals for a comprehensive and holistic diagnosis .
\end{abstract}

Key words: HIVIAIDS, nursing care, CSRA method.
Correspondencia: Patricia Larios-López

Email: patricia_colegio@yahoo.com.mx

Artículo recibido: 22 de octubre de 2021

Artículo aceptado: 15 de noviembre de 2021
Carrera-Cisneros AD y Larios-López P. Modelo de "análisis de resultados del estado actual" (AREA) en un paciente con VIH/ SIDA para el plan de cuidados de enfermería. Caso clínico Caso clínico. CyRS. 2021; 3(2):26-35

DOI: https://doi.org/10.22201/fesz.26831422e.2021.3.2.4 


\section{INTRODUCCIÓN}

El síndrome de inmunodeficiencia adquirida (SIDA) causado por el virus de inmunodeficiencia humana $(\mathrm{VIH})$, se ha convertido en epidemia mundial y principal causa infecciosa de muerte en adultos jóvenes en el mundo. De acuerdo con ONUSIDA en el año 2020 , más de 37.7 millones de personas vivían con el $\mathrm{VIH}$, de las cuales 36 millones son adultos; cerca de 1.5 millones contrajeron la infección en el 2020; y alrededor de 680,000 millones murieron a causa de enfermedades relacionadas con el SIDA. En lo que respecta a América Latina, en 2019 existían 2.1 millones de personas con VIH, hubo más de 100,000 nuevas infecciones y cerca de 31,000 muertes relacionadas con el SIDA. ${ }^{1}$ En México la Secretaría de Salud reporta qué de 1983, año en el que se presentó el primer caso en el país, al 2021 se han notificado 322,987 personas con $\mathrm{VIH} ; 195,860$ se encuentran vivas. Así mismo, el Centro Nacional para la Prevención y Control del VIH y el sida (CENSIDA) señaló que hasta mayo del 2021 se registraron 113,788 personas en Tratamiento con Antirretrovirales en la plataforma del Sistema de Administración, Logística y Vigilancia de Antirretrovirales (SALVAR) de la Secretaría de Salud, de los cuales 9,638 casos se encuentran en la ciudad de México. ${ }^{2}$ Por otro lado, a finales del 2020, había 21,500 millones de dólares disponibles para la atención de la enfermedad en los países de ingresos bajos y medianos; se estima que en 2025 se necesitarán 29,000 millones para su atención. Todo lo anterior muestra la seria amenaza que representa el VIH para la salud pública mundial y la necesidad de fortalecer programas para la prevención, detección temprana y perfeccionar el tratamiento de la enfermedad y de la calidad de la atención. ${ }^{2-4}$

El VIH pertenece a un grupo viral conocido como retrovirus. Este virus causante de la enfermedad se divide en familias como: Oncoviridae, Espumaviridae y los Lentiviridae, causando esta última inmunodeficiencia, logrando afectar y destruir a las células que infectan; provocando el $\mathrm{VIH}-1$ y 2 , analizados por primera vez en 1983 y 1986, respectivamente. Los virus son parásitos intracelulares y todos tienen como objetivo células específicas, el VIH lo tiene en células con receptores CD4, los cuales se encuentran en la superficie de linfocitos y monocitos, de ahí que, una vez que el VIH se fija a la célula hospedadora el virus se replica y causa una disminución lenta y continua de los linfocitos. ${ }^{5}$
EL VIH-1 se transmite en los líquidos corporales que incluyen la sangre, líquido seminal, secreciones vaginales, líquido amniótico y leche materna. La infección evoluciona en cuatro fases: (i) fase de infección aguda, en la que ingresa el virus al cuerpo, caracterizada por dos estadios, el primero puede ser asintomático y el segundo con síntomas como fiebre, linfadenopatias, faringitis, cuadros similares a la mononucleosis, mialgia, artralgias, anorexia, pérdida de peso, erupciones cutáneas, alopecia, urticaria, náuseas, vómito, diarrea, cefalea, dolor y otros padecimientos; (ii) fase asintomática, en la que después de alcanzar la carga viral estable, las personas entran en una etapa crónica en la cual el sistema inmunitario no puede eliminar el virus, esta fase varia de un paciente a otro y dicta la velocidad de progresión de la enfermedad; pueden pasar en promedio de 8 a 10 años antes que la persona presente una complicación importante; (iii) fase sintomática de la infección de VIH, en la que con el paso de los meses o años, según la calidad del sistema inmunológico, se empiezan a desarrollar síntomas como síndrome febril, anemia, tos seca y persistente, así como diarreas que pueden durar más de un mes, candidiasis bucofaríngea, vulvovaginitis, verrugas genitales o anales, herpes zoster, meningitis aséptica y estados depresivos, entre otros; (iv) fase de Síndrome de Inmunodeficiencia Adquirida (SIDA), es la etapa final en la que se caracteriza por la aparición de infecciones oportunistas y tumores extraños; existe una inmunodepresión severa derivada de la disminución de linfocitos $\mathrm{CD}^{+}$, causando un importante deterioro del sistema inmunológico comprometiendo la vida del paciente..$^{5,6}$

Las personas con VIH/SIDA presentan varios síntomas relacionados con la enfermedad, efectos secundarios a los antiretrovirales y de otras enfermedades. Infecciones, neoplasias malignas o el efecto directo en los tejidos corporales son trastornos relacionados con la infección. También existe un alto riesgo de desarrollar múltiples complicaciones asociadas con fenómenos de inmunosenescencia no relativos a la infección per se, entre ellas resistencia insulínica, síndrome metabólico, neurodegeneración, alteraciones cognitivas, esteatohepatitis no alcohólica, osteoporosis, insuficiencia renal y enfermedades autoinmunes, así como aterosclerosis acelerada y enfermedad cardiovascular. ${ }^{7,8}$ 
La mejora en la atención y la accesibilidad a los antirretrovirales ha incrementado de manera significativa la esperanza de vida de las personas con el $\mathrm{VIH} /$ SIDA, convirtiéndolo en una enfermedad crónica compleja pues el diseño de regímenes de tratamiento individualizados y óptimamente efectivos para cada paciente se ha convertido en un desafío. ${ }^{9,10}$ Por otro lado, una sólida adherencia al tratamiento resulta vital para retardar la progresión de la enfermedad, disminuir la carga viral circulante, restaurar el sistema inmunológico y mejorar la calidad de vida. Desafortunadamente su complejidad hace que muchos pacientes se cansen, generando poca adherencia e inclusive abandonen el tratamiento; más de $80 \%$ de los enfermos de VIH/SIDA fallecen severamente inmunodeprimidos, padeciendo múltiples infecciones oportunistas y neoplasias en extremo agresivas. ${ }^{11}$ En este contexto clínico el plan de cuidados de enfermería es una herramienta fundamental para contribuir en el diagnóstico temprano de complicaciones, para el logro de un tratamiento integral, mejorar el pronóstico y en algunos casos acompañar en una muerte digna a los pacientes con dicha enfermedad.

En la planificación de la atención de enfermería se centra en identificar las respuestas humanas y la capacidad de la persona para funcionar de forma independiente, para ello, hace uso de su juicio diagnóstico (fases de valoración y diagnóstico) y juicio terapéutico (planificación, ejecución y evaluación de las actividades realizadas). ${ }^{12}$ Así como de un leguaje estandarizado, específicamente el de los criterios establecidos por la Asociación Norteamericana de Diagnóstico de Enfermería (NANDA, North American Nursing Diagnosis Association), la Clasificación de Resultados de Enfermería (NOC, Nursing Outcomes Classification) y la Clasificación de Intervenciones de Enfermería (NIC, Nursing Interventions Classification). ${ }^{13-15}$

Otra herramienta utilizada es el Modelo de Análisis de Resultados del Estado Actual (AREA), el cual establece una estructura para el razonamiento clínico que se centra en los resultados esperados en la práctica de enfermería. Es de suma utilidad en casos con múltiples diagnósticos, donde el equipo de enfermería debe realizar una hipótesis diagnóstica y clínica, tanto de forma autónoma como interdependiente que facilite el enmarcar, yuxtaponer, probar y emitir juicios sobre la transición desde el estado actual hacia el resultado esperado. ${ }^{12}$ Sin embargo, este no siempre se realiza en la práctica clínica, por lo que el objetivo de este trabajo es realizar un análisis crítico del plan de cuidados de enfermería convencional y presentar la comparación con el modelo AREA de razonamiento clínico de un caso de VIH/SIDA con múltiples complicaciones.

\section{Caso cLínICO}

Paciente masculino de 57 años, casado que vive con su esposa en la Ciudad de México. Actualmente padece VIH/SIDA y hepatitis $B$, con 25 años de evolución; patologías resultantes de prácticas sexuales riesgosas, admitió no haber usado condón con su esposa y con ninguna otra de sus parejas. Además, con un gran desapego al tratamiento médico para el control de ambas patologías. Posteriormente desarrollo diabetes mellitus tipo 2 controlada; además de antecedente de un infarto agudo al miocardio. En las notas de seguimiento del paciente por enfermería se reportó lo siguiente:

\section{NOVIEMBRE 2019}

El paciente acude al servicio de urgencias con dolor abdominal. Se indican exámenes de laboratorio y resonancia magnética encontrándose una colecistitis crónica litiásica. El paciente fue preparado para intervención quirúrgica con glucemia capilar de $150 \mathrm{mg} /$ dL, FR.20/minuto, FC.76/minuto y TA de 135/90 $\mathrm{mmHg}$. Posteriormente, es trasladado al servicio de cirugía general por una colecistectomía complicada donde se detectó hígado macro-nodular que puede ser pos-viral, por virus B. Además, con instalación de sonda en T a derivación y catéter vesical.

\section{DE NOVIEMBRE 2019}

El paciente en su primer día de recuperación se encuentra alerta, en cama sin barandales, en ayuno, sin datos de infección en herida quirúrgica, sin dolor y con úlcera por presión (UPP) grado II en zona sacra. La lista de medicamentos se presenta en el cuadro 1. Signos vitales: TA: $110 / 70 \mathrm{mmHg}$, FC.76/ min, FR. $16 / \mathrm{min}$, Temperatura $36.5^{\circ} \mathrm{C}$.

\section{DE NOVIEMBRE 2019}

El paciente presenta dolor en región abdominal irradiándose al hipocondrio derecho, acompañado de náuseas y vómito. Se encuentra sin sonda T. Gasometría arterial: PCO2: $32 \mathrm{mmHg}$, HCO3: 20-15 $\mathrm{mEq} / \mathrm{L}, \mathrm{PH}:$ 7.19. 


\section{Cuadro 1. Tratamiento Médico}

\begin{tabular}{cl}
\hline 8 de noviembre & $\begin{array}{l}\text { MEDICAMENTOS } \\
\text { Tratamiento médico de inicio: }\end{array}$ \\
\hline 1 & Omeprazol $40 \mathrm{mg}$. cada 12 horas IV. \\
2 & Truvada 200mg-245 mg. comprimidos cada 24 horas por SNG \\
3 & Norvir comprimidos de $100 \mathrm{mg}$. cada 24 horas por SNG \\
4 & Raltegravir tableta $400 \mathrm{mg}$. cada 12 horas por SNG \\
5 & $\begin{array}{l}\text { Solución Hartman } 1000 \mathrm{ml} \text {. pasar en } 12 \text { horas IV. } \\
\text { Buprenorfina solución inyectable, infusión } 900 \mathrm{mg} . \text { en } 250 \mathrm{cc} . \text { de } \\
\text { solución cloruro de sodio. }\end{array}$ \\
\hline
\end{tabular}

\section{2 de noviembre Se anexa a las indicaciones lo siguiente:}

7

8

9
Midazolam $105 \mathrm{mg}$. en $100 \mathrm{ml}$. en infusión continúa pasar $5 \mathrm{ml}$. por hora

Norepinefrina 8 mg. solución intravenosa en 250cc. de solución fisiológica, pasar a $8 \mathrm{ml} /$ hora.

Octreotide $5 \mathrm{mg}$. en $5 \mathrm{ml}$. cada 8 horas IV.

SNG: Sonda Nasogástrica; IV: Intra Venoso

\section{DE NOVIEMBRE 2019}

El paciente presenta deshidratación, con balances de líquidos negativos, úlceras por presión grado II en región sacra y tobillos. Se indica toma de laboratorios y RX de tórax.

En hoja de enfermería se registra el diagnóstico: riesgo de ulcera por presión con actividades de cambio de posición cada dos horas.

\section{DE NOVIEMBRE 2019}

El paciente presenta acidosis respiratoria y sangrado hepático, a la interpretación radiológica se encuentra problema en el parénquima pulmonar, con saturación de 02 menor al $60 \%$, se procede a la intubación y manejo de ventilación mecánica invasiva, sedación con midazolam $105 \mathrm{mg}$. en solución cloruro sodio $100 \mathrm{ml}$. infusión continua (cuadro 1). Se mantiene en ayuno, con balances negativos superiores de $100 \mathrm{ml}$. por turno. Signos vitales: T/A. 90/60 mmHg, FC.58/ minuto, FR.20/minuto, temperatura $37^{\circ} \mathrm{C}$.

\section{DE NOVIEMBRE 2019}

El paciente continúo con balances de líquidos negativos, glucemia sin alteración. Signos vitales: T/A. $70 / 40 \mathrm{mmHg}$, FC.58/minuto, FR.20/minuto y temperatura $37^{\circ} \mathrm{C}$.

En hoja de enfermería se registran tres diagnósticos en este orden: riesgo de caídas, úlceras por presión y patrón respiratorio ineficaz; con registro de monitorización de la respiración y movilización del paciente cada dos horas.

\section{DE NOVIEMBRE 2019}

El paciente continúa con ventilación mecánica, se realiza aspiración de secreciones oro-traqueales las cuales fueron abundantes, fétidas y espesas, con catéter vesical a derivación y balance de líquidos negativos a $1200 \mathrm{ml}$ de pérdidas en 24 horas. Se continuó con control de líquidos, curación de herida quirúrgica y movilización cada dos horas. Se instaló sonda nasogástrica a derivación. Signos vitales: T/A. 90/60 mmHg, FC.58/minuto, FR.20/minuto, Temperatura $37^{\circ} \mathrm{C}$. 


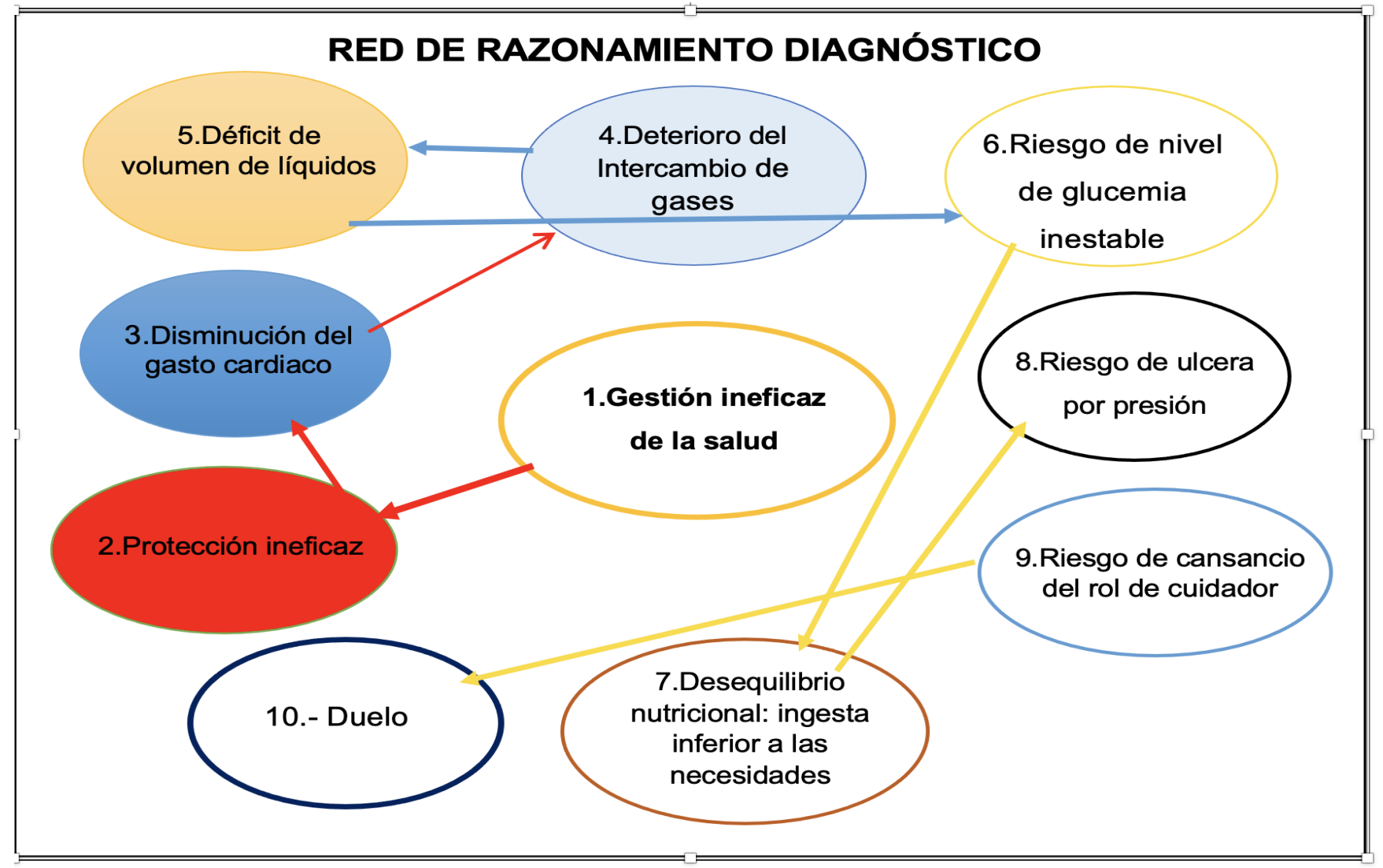

Figura 1. Red de Razonamiento Diagnóstico (ws) útil donde existen más de tres diagnósticos médicos que van complicando el estado de salud hasta la muerte del paciente. Permite visualizar las relaciones entre un gran número de diagnósticos (NANDA) y las innumerables posibilidades que se presentan para su priorización.

\section{NOVIEMBRE 2019}

El paciente fallece 12:35 pm con el diagnóstico de defunción por neumonía nosocomial. Es importante mencionar que desde su ingreso al servicio de cirugía general compartió cubículo con 7 pacientes y sus respectivos familiares.

\section{ANÁLISIS CRÍTICO UTILIZANDO EL MÉTODO AREA}

Los cuidados de enfermería se centraron en el mantenimiento de la ventilación mecánica, monitorización de signos vitales, control de líquidos y movilización cada dos horas. Además, se identifican varios problemas de salud que requieren un diagnóstico de enfermería, situación compleja porque es necesario resolver varios problemas a la vez. De ahí que una opción para estos casos es el uso del método AREA para la integración y análisis de una red de razonamiento diagnóstico, utilizando las taxonomías NANDA, NOC y NIC en la priorización del cuidado.
Para este caso se realizó una red de razonamiento diagnóstico (figura 1) en la que se identificaron tres diagnósticos prioritarios: (i) "GESTIÓN INEFICAZ DE LA SALUD", debido a que el paciente mostró un gran desapego al tratamiento médico; además de prácticas sexuales riesgosas causantes del VIH/SIDA y hepatitis B; (ii) "PROTECCIÓN INEFICAZ", debido a los padecimientos asociados con el VIH/SIDA; .(iii) "DISMINUCIÓN DEL GASTO CARDÍACO", correspondiente a la sintomatología en el estadio más grave del paciente referido del 13 al 17 de noviembre del 2019 (cuadros 2-4).

La red de razonamiento diagnóstico en red permite identificar prioridades en la atención del paciente, así como una las actividades que es necesario realizar para un cuidado adecuado del paciente. De entre ellas, es de resaltar las actividades de control de infecciones, las cuáles no se realizaron en el caso presentado. 
Cuadro 2. Diagnóstico enfermero primario

\section{Plan de cuidados}

\begin{tabular}{|c|c|c|c|c|c|c|c|}
\hline Servicio & Cama & Sala & \multicolumn{5}{|c|}{ Clasificación de los resultados de enfermería (noc) } \\
\hline \multicolumn{3}{|c|}{$\begin{array}{l}\text { Gestión ineficaz de la } \\
\text { propia salud (00078) }\end{array}$} & \multicolumn{2}{|c|}{$\begin{array}{l}1813 \text { DOMINIO: conocimiento y } \\
\text { conducta de salud }\end{array}$} & \multicolumn{3}{|c|}{$\begin{array}{l}\text { CLASE: conocimiento sobre } \\
\text { conducta de salud }\end{array}$} \\
\hline & & & $\begin{array}{l}\text { Resultado } \\
\text { esperado }\end{array}$ & Indicadores & & & \\
\hline \multirow{3}{*}{\multicolumn{3}{|c|}{$\begin{array}{l}\text { Gestión ineficaz de la } \\
\text { propia salud R/C conoci- } \\
\text { mientos insuficientes del } \\
\text { régimen terapéutico M/P } \\
\text { fracaso al incluir el régi- } \\
\text { men terapéutico en la vida } \\
\text { diaria. }\end{array}$}} & \multirow{3}{*}{$\begin{array}{l}\text { (1813) Cono- } \\
\text { cimiento: régi- } \\
\text { men terapéutico }\end{array}$} & $\begin{array}{l}\text { (181310) Proceso } \\
\text { de la enferme- } \\
\text { dad }\end{array}$ & \multicolumn{3}{|c|}{$\begin{array}{l}\text { Escala de medición } \\
\qquad \text { puntuación diana }\end{array}$} \\
\hline & & & & $\begin{array}{l}181311) \text { Benefi- } \\
\text { cios del tratami- } \\
\text { ento }\end{array}$ & \multirow{2}{*}{$\begin{array}{l}\text { Ningún conocimiento } \\
\text { Conocimiento escaso } \\
\text { Conocimiento moderado } \\
\text { Conocimiento sustancial } \\
\text { Conocimiento extenso }\end{array}$} & \multirow{2}{*}{$\begin{array}{l}1 \\
2 \\
3 \\
4 \\
5\end{array}$} & \\
\hline & & & & $\begin{array}{l}\text { (181302) Respon- } \\
\text { sabilidad del au- } \\
\text { tocuidado para } \\
\text { el tratamiento en } \\
\text { curso }\end{array}$ & & & $\begin{array}{l}2 \\
0\end{array}$ \\
\hline
\end{tabular}

\section{Cuidados cardiacos (1620)}

Actividades
Actividades

1. Determinar las necesidades de enseñanza del paciente

2. Determinar la preparación del paciente para aprender

3. Valorar el nivel actual de conocimientos y compresión de contenidos del paciente

4. Determinar la capacidad del paciente para asimilar información especifica

5. Determinar la motivación del paciente para asimilar información especifica

6. Ajustar la instrucción para facilitar el aprendizaje Instruir al paciente cuando corresponda

7. Instruir al paciente cuando corresponda

8. Corregir malas interpretaciones de información según corresponda

9. Remitir al paciente con especialista/centros para reforzar el conocimiento.
1. Establecer metas realistas de aprendizaje para el paciente

2. Establecer la información en una secuencia lógica

3. Proporcionar la información de sencilla a compleja, desconocida a conocida, según corresponda

4. Adaptar la información para que cumpla con el estilo de vida o rutina del paciente

5. Relacionar la información con las necesidades y deseos del paciente

6. Proporcionar información de acuerdo con la situación de control del paciente

7. Simplificar las instrucciones siempre que sea posible

8. Repetir la información importante

9. Asegurarse que la información proporcionada por los diferentes miembros del equipo de salud sea congruente.

\section{Abreviaturas: NANDA, North American Nursing Diagnosis Association; NOC, Nursing Outcomes Classification; NIC, Nursing} Interventions Classification 


\section{Cuadro 3. Diagnóstico enfermero secundario}

\section{Plan de cuidados}

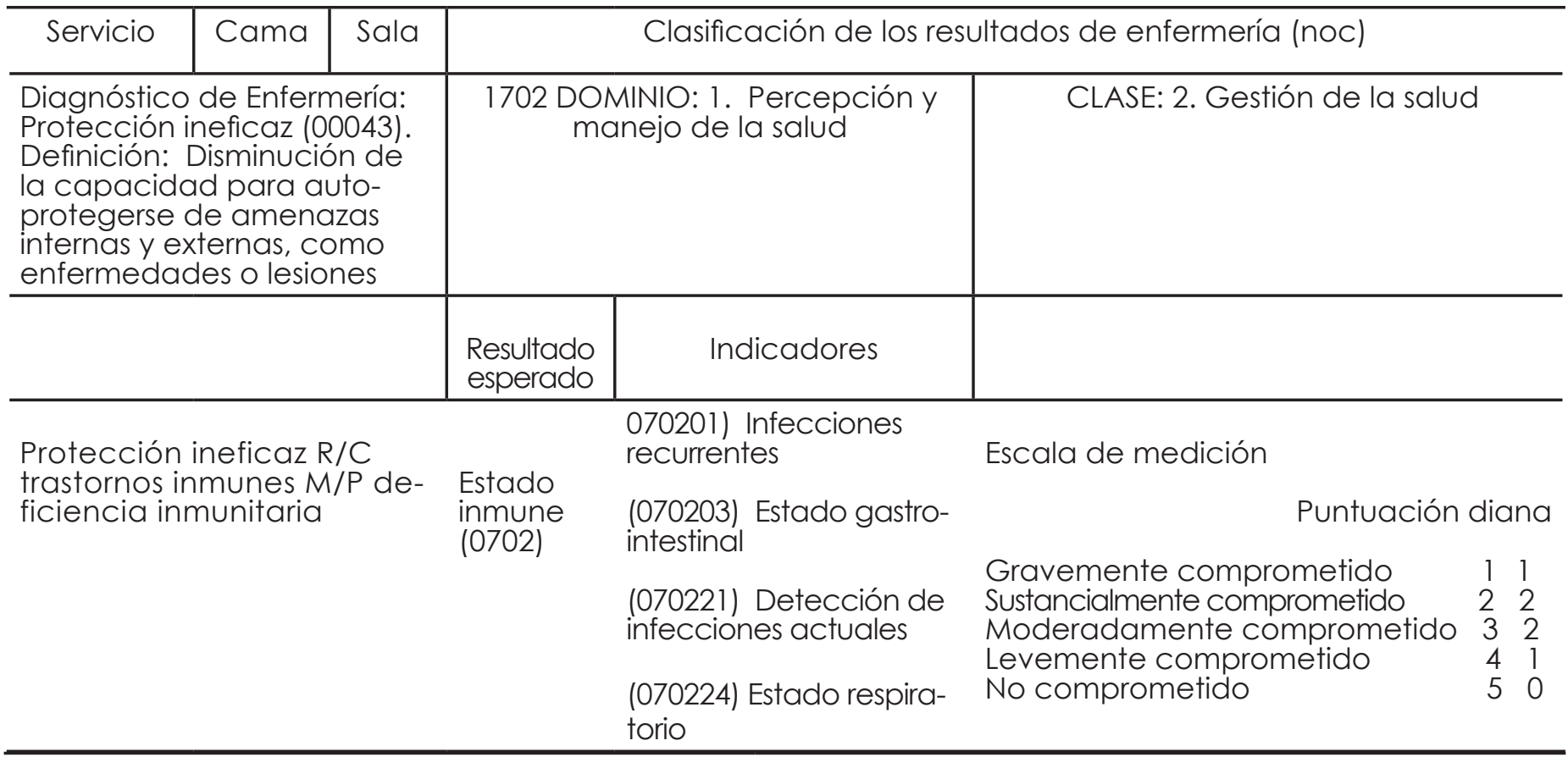

Control de infecciones (6540) Actividades

\section{Manejo de la vía aérea artificial (3140)}

Actividades
1. Limpiar el ambiente adecuadamente después de cada uso por parte de los pacientes

2. Cambiar el equipo de cuidados del paciente según protocolo del centro

3. Aislar a las personas expuestas a enfermedades transmisibles

4. Mantener técnica de aislamiento

5. Poner en práctica precauciones universales

6. Mantener un ambiente séptico mientras se

cambian los sistemas, incluyendo la nutrición parenteral

7. Administrar terapia de antibióticos

8. Garantizar una manipulación aséptica de todas las líneas IV

9. Controlar las condiciones que indican la necesidad de soporte ventilatorio
1. Observar si hay insuficiencia respiratoria inminente

2. Asegurar que las alarmas del ventilador estén conectadas

3. Comprobar de forma regular las conexiones del ventilador

4. Controlar los factores que puedan aumentar el trabajo respiratorio del paciente

5. Vigilar la eficacia de la ventilación mecánica sobre el estado fisiológico

6. Monitorizar los efectos de la ventilación sobre la saturación de oxigeno

7. Registrar el color cantidad y consistencia de las secreciones aspiradas

8. Documentar los cambios de ajustes del ventilador

9. Mantener una técnica totalmente aséptica.

Abreviaturas: NANDA, North American Nursing Diagnosis Association; NOC, Nursing Outcomes Classification; NIC, Nursing Interventions Classification 


\title{
Cuadro 4. Diagnóstico enfermero terciario
}

\section{Plan de cuidados}

\begin{tabular}{l|c|c|c}
\hline Servicio & Cama & \multicolumn{1}{c|}{ Sala } & \multicolumn{2}{c}{ Clasificación de los resultados de enfermería (noc) } \\
\hline $\begin{array}{l}\text { Diagnóstico de Enfermería: Disminu- } \\
\text { ción del Gasto Cardiaco (00029). }\end{array}$ & $\begin{array}{c}\text { 1401 DOMINIO: 4 Actividad y re- } \\
\text { poso }\end{array}$ & $\begin{array}{c}\text { CLASE: 4 Respuestas cardio- } \\
\text { vasculares pulmonares }\end{array}$ \\
$\begin{array}{l}\text { Definición: la cantidad de sangre } \\
\text { bombeada por el corazón es inade- } \\
\text { cuada para satisfacer las demandas } \\
\text { metabolicas del cuerpo. }\end{array}$ & & \\
\hline $\begin{array}{l}\text { Disminución del Gasto Cardiaco R/C } \\
\text { alteraciones del ritmo cardiaco M/P } \\
\text { bradicardia y alteraciones de la pre- } \\
\text { sión arterial. }\end{array}$ & $\begin{array}{c}\text { Resultado } \\
\text { esperado }\end{array}$ & Indicadores & \\
\hline
\end{tabular}

Gestión ineficaz de la propia salud $\mathrm{R} / \mathrm{C}$ conocimientos insuficientes del régimen terapéutico M/P fracaso al incluir el régimen terapéutico en la vida diaria.
Efectividad de la (0400019) Presión sanbomba guínea diastólica cardiaca (0400).

(040002) Frecuencia
(040001) Presión sanguínea sistólica cardiaca

(040006) Pulsos periféricos
Escala de medición

puntuación diana

Desviación grave

Desviación sustancial

Desviación moderada

Desviación leve

Sin desviación

$\longrightarrow$

\section{Enseñanza: individual (5606)}

\section{Facilitar el aprendizaje (5520)}

\author{
Actividades
}

Actividades

1. Monitorizar al paciente desde los puntos de vista físico, psicológico, según las normas del centro

2. Garantizar un nivel d actividad que no comprometa el gasto cardiaco y que no provoque crisis cardiacas.

3. Monitorizar los signos vitales con frecuencia FC, FR, Presión arterial.

4. Monitorizar el estado cardiovascular

5. Monitorizar la aparición de arritmias cardiacas, incluidos los trastornos de ritmo como de conducción 6. Monitorizar los cabios del segmento ST en el EKG según corresponda

7. Realizar una valoración exhaustiva de la circulación periférica (comprobar pulsos periféricos, edema, relleno capilar, color y temperatura de las extremidades.

8. Observar los signos y síntomas de disminución del gasto cardiaco.

9. Monitorizar el estado respiratorio, por si aparecen síntomas de insuficiencia cardiaca.
1. Monitorizar el equilibrio hídrico (entradas y salidas, Peso diario).

2. Monitorizar si los valores de laboratorio son correctos, (enzimas cardiacas y niveles de electrolitos).

3. Evaluar las alteraciones de la presión arterial 4. Monitorizar la aparición de disnea, fatiga, taquipnea y ortopnea.

5. Establecer una relación de apoyo con el paciente y la familia.

Abreviaturas: NANDA, North American Nursing Diagnosis Association; NOC, Nursing Outcomes Classification; NIC, Nursing Interventions Classification 


\section{Discusión}

La atención a la pandemia del VIH/SIDA ha sido un reto para el sector salud, debido a su alta prevalencia, el incremento de la atención médica y los costos del tratamiento. El incremento de la expectativa de vida de las personas infectadas con el VIH/SIDA permite que se considere como una enfermedad crónica en la que un adecuado seguimiento puede mejorar su calidad de vida a lo largo de la enfermedad. . $^{416}$

El cuidado de la experiencia de la salud humana es una tarea de la enfermería, para realizarla se ha organizado y sistematizado el proceso de diagnóstico y tratamiento que le permita afrontar los problemas reales y potenciales del paciente. Por tal motivo, es necesario que la enfermera o enfermero posea los conocimientos sobre la enfermedad, las habilidades para realizar procedimientos y un pensamiento crítico que se ajuste a las situaciones que se le presenten. ${ }^{17}$

El proceso de diagnóstico en enfermería es el eje metodológico para brindar cuidados, se desarrolla en cinco etapas (valoración, diagnóstico, planeación, ejecución y evaluación) que permiten seguir el estado de salud del paciente de forma objetiva. A este esquema se ha incorporado paulatinamente el conocimiento proporcionado por investigaciones científicas, el considerar al paciente en el entorno en que se encuentra e identificar el resultado actual, el obtenido y el esperado. ${ }^{18}$

En padecimientos complejos como en el caso presentado con VIH/SIDA descontrolado con polipatología es necesario identificar el problema prioritario, búsqueda de información sistematizada, análisis crítico de la evidencia científica, uso de la taxonomía enfermera y la aplicación del método AREA como modelo de diagnóstico en red, que permite un análisis integral y holístico, tal como se muestra en el caso clínico presentado. En este sentido, es muy probable que los cuidados de enfermería con el modelo AREA, influyan en la evolución clínica de este tipo de pacientes, pero sobre todo, tendrá un impacto en calidad de la atención y confort para el paciente. Además, de identificar el perfil de la persona hospitalizada, los cuidados paliativos, las condiciones que intervienen, para la gestión del cuidado, las condiciones que intervienen, y aspectos para organizar y administrar el cuidado. ${ }^{19,20}$
La red de razonamiento diagnóstico del modelo AREA permite a los profesionales de enfermería la priorización de intervenciones en casos complejos como en pacientes con VIH/SIDA. Esta herramienta facilita aplicar y desarrollar habilidades críticas, metacognitivas y de pensamiento crítico. ${ }^{18,21}$ Bajo este contexto, los enfermeros y enfermeras deben tener la habilidad de adaptarse a las diferentes coyunturas históricas, como es el caso de los actuales cambios en el sistema de salud, que demanda a los profesionales múltiples capacidades para hacer frente a un incremento de responsabilidades. Asimismo, las Instituciones Educativas también de incluir en los planes de estudio contenido y prácticas clínicas. Así como modelos orientados garantizando la formación en las buenas prácticas. orientados a la formación en las buenas prácticas clínicas, promoviendo el desarrollo de profesionales analíticos y críticos con capacidad de actuación ante los complejos escenarios de la salud en México.

\section{CONCLUSIÓN}

La elaboración de un Plan de Cuidados de Enfermería individualizado, aplicando el método AREA como herramienta para la red de razonamiento diagnóstico. Lo anterior, promueve la reflexión la red de razonamiento diagnóstico, promueve la reflexión crítica en los profesionales de Enfermería y garantiza el cuidado en pacientes con enfermedades complejas como el VIH/SIDA, con lo cual se mejora la calidad de la atención y el confort en este tipo de pacientes.

\section{Agradecimientos}

Agradecemos el apoyo del Dr. José Miguel Sánchez Nieto por la edición del artículo. El manuscrito fue revisado y editado en el Programa para la Investigación Bibliográfica Científica sobre Salud (PIBCIS) de la FES Zaragoza, UNAM

\section{Referencias}

1. ONUSIDA. Hoja informativa 2021. 2021. [Internet] Ginebra: ONUSIDA; 2021. [Consultada: 20 de octubre de 2021]. Disponible en: https://www.unaids.org/ sites/default/files/media_asset/UNAIDS_FactSheet_ es.pdf 
2. CENSIDA. Boletín de atención integral de personas que viven con $\mathrm{VIH}$. [Internet] México: Secretaría de Salud; 2021. [Consultada: 18 de octubre de 2021]. Disponible en:

https://www.gob.mx/cms/uploads/attachment/ file/670963/Bol_DAI_Vol7_N3_SEP2021.pdf

3. CENSIDA. Guía de manejo antirretroviral de las personas con VIH 2021. [Internet] México: Secretaría de Salud; 2021. [Consultada: 18 de octubre de 2021]. Disponible en:

http://www.csg.gob.mx/descargas/pdf/priorizacion/ guias/Guia_ARV_170821.pdf

4. Franco BCA, Estrada MJH. Impacto macroeconómico generado por la pandemia del VIH/SIDA informado por la literatura internacional desde 1990 a 2013. Acta Odontológica Colombiana. 2018; 8(2):72-97.

5. Kasper D. Harrison: Principios de Medicina Interna. México: McGraw Hill; 2006.

6. Hinkle JL, Cheever KH. Brunner y Suddarth. Enfermería medicoquirúrgica. $12^{\mathrm{a}}$ ed. Philadelphia: Wolters Kluwer; 2012.

7. Chan-Cheng HC, Monge-Rodríguez SL, EspinozaMora MR. Activación inmunológica crónica en la patogénesis de la enfermedad por virus de inmunodeficiencia humana. Acta Méd. Costarric. 2017; 59(1): 7-14.

8. Webel AR, Schexnayder J, Cioe PA, Zuñiga JA. A review of chronic comorbidities in adults living with HIV: state of the science. J Assoc Nurses AIDS Care. 2021; 32(3):322-346. doi: 10.1097/ JNC.0000000000000240.

9. Ghosn J, Taiwo B, Seedat S, Autran B, Katlama C. HIV. Lancet. 2018; 392(10148): 685-697. doi. org/10.1016/S0140-6736(18)31311-4

10. Kumbale CM, Voit EO. Hacia una medicina personalizada para el VIH/SIDA.J AIDS HIV Treat. 2021; 3 (2): $37-41$.

11. Betancourt-Gambino J. Adherencia al tratamiento antirretroviral en pacientes seropositivos. Rev Cubana Med Gen Integr. 2018; 34(3): 82-93.
12. Rubio Sevilla JC. Papel de enfermería en el juicio clínico: la valoración y el diagnóstico. Enfermería en Cardiología. 2014; 21 (62): 25-31

13. Herdman $H$, Shigemi K. NANDA International diagnósticos enfermeros definiciones y clasificación. $11^{\mathrm{a}}$ ed. Barcelona: Elsevier; 2018-2020.

14. Moorhead S, Johnson M, Maas M, Swanson E. Clasificación de resultados de enfermería NOC medición de resultados en salud. $6^{a}$ ed. Barcelona: Elsevier; 2019.

15. Bulechek M, Dochterman J, Butcher K, Wagner C. Clasificación de intervenciones de enfermería NIC. $7^{\text {a }}$ ed. Barcelona: Elsevier; 2019.

16. Cardona-Arias A, Higuita-Gutiérrez LF. Impacto del VIH/SIDA sobre la calidad de vida: metaanálisis 2002-2012. Revista Española de Salud Pública. 2014; 88(1):87-101.

17. Reina NC. El proceso de enfermería: instrumento para el cuidado. Umbral Científico. 2010; (17): 18-23.

18. González-Castillo MG, Monroy-Rojas A. Proceso enfermero de tercera generación. Enfermería Universitaria. 2016; 13(2):124-129.

19. López-Morales AB, Barrera-Cruz A, Alarcón-Morales $\mathrm{C}$, Martínez-Ravelo $\mathrm{R}$. Enfermería basada en la evidencia: plan de cuidados para pacientes con $\mathrm{VIH} /$ SIDA (parte 1). Revista de Enfermería del Instituto Mexicano del Seguro Social. 2016; 24(3): 229-233.

20. Zepeda KGM, da Silva MM, Dos Santos DCL, Gaspar RB, Trotte LAC. Management of nursing care in HIVIAIDS from a palliative and hospital perspective. 2019 ; 72(5): 1243-1250. doi: 10.1590/0034-71672017-0431.

21. Alfaro LR. Aplicación del Proceso Enfermero, Fundamento del Razonamiento Clínico. $8^{\mathrm{a}}$ ed. Londres: Wolters Kluwer; 2014. 